\title{
Insuficiencia mitral severa asintomática: ¿intervención quirúrgica o seguimiento clínico-ecocardiográfico?
}

Viñeta clínica

Paciente de sexo femenino, 39 años, dos hijos. Portadora de insuficiencia mitral que controla anualmente. Sin historia de disnea. En el último control ecocardiográfico se informa: diámetro telesistólico de ventrículo izquierdo: $44 \mathrm{~mm}$ (previo $40 \mathrm{~mm}$ ); diámetro de aurícula izquierda: $40 \mathrm{~mm}$; fracción de eyección de ventrículo izquierdo: 60\% (previa 66\%). Válvula mitral mixomatosa con prolapso de ambos velos que determina una insuficiencia severa.

\section{Por qué realizar cirugía precoz Dr. Gerardo Soca}

\section{Introducción}

Estamos frente a una paciente joven que padece insuficiencia mitral primaria, crónica, severa y asintomática, con fracción de eyección de ventrículo izquierdo (FEVI): 60\%, diámetro sistólico de ventrículo izquierdo (DSVI): 44 mm y diámetro de aurícula izquierda (AI): $40 \mathrm{~mm}$.

Se aportan datos relevantes del ecocardiograma del año anterior: FEVI 66\%, DSVI 40mm. El mecanismo de la insuficiencia fue definido como prolapso bivalvar.

\section{Evaluación complementaria}

Para complementar la evaluación de una insuficiencia mitral (IM) severa y asintomática son útiles estudios de imagen y funcionales. Entre los primeros destaca el ecocardiograma transesofágico (ETE) para determinar la anatomía valvular precisa y el mecanismo de la insuficiencia, fundamentalmente en vistas a una eventual reparación. La ecografía 3D, disponible en Uruguay, es otra herramienta de gran valor en el preoperatorio, ya que permite una mejor planificación de la estrategia quirúrgica.

Desde el punto de vista funcional, la IM asintomática debería evaluarse con una prueba ergométrica para determinar la capacidad funcional y real ausencia de síntomas, (indicación IIa, nivel de evidencia $\mathrm{B})^{(1)}$. Desconocemos nivel de actividad, aunque estimamos que sea elevado.

Otro estudio funcional útil en este escenario es la ecografía de ejercicio. Permite evaluar cambios sobre la presión arterial pulmonar (PSAP) y la FEVI, ambas de gran valor pronóstico en IM asintomática. Según un estudio observacional en el que se siguió ecocardiográficamente a pacientes con IM

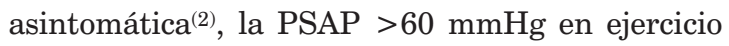
presenta un valor pronóstico adverso, aún mayor que en reposo. Estos pacientes tuvieron menor probabilidad de permanecer asintomáticos a dos años en comparación con los que no alcanzaron esa PSAP con ejercicio ( $35 \pm 8 \%$ vs $75 \pm 7 \%, \mathrm{p}<0,0001)$. Este dato podría alentar a intervenir precozmente a una paciente tan joven (en la mitad de la expectativa de vida general de mujeres en Uruguay).

Si la presión pulmonar es el único determinante para enviar a cirugía mitral a un paciente con IM asintomática, este dato debería confirmarse mediante medida invasiva por cateterismo derecho, dado que define la indicación quirúrgica per se.

La resonancia cardíaca es un excelente método anátomo-funcional para determinar la severidad de la insuficiencia en los casos en que el grado de esta es dudoso. Determina muy precisamente la FEVI y 
los diámetros ventricular y auricular izquierdos. Estos parámetros son determinantes en la definición de la conducta en un paciente con IM asintomática; por tanto, su medición precisa es fundamental ${ }^{(3)}$.

\section{Cuándo intervenir una insuficiencia mitral asintomática}

Sobre la discusión de indicación quirúrgica, tomaremos como eje las guías de valvulopatías 2017 de la AHA/ACC y ESC ${ }^{(1,4)}$.

En las guías europeas se postula que FEVI $\leq 60 \%$, DSVI $\geq 45 \mathrm{~mm}$, presencia de FA o PSAP $>50 \mathrm{mmHg}$ se relacionan con peor evolución a largo plazo después de cirugía (independientemente del estado sintomático previo) y deben considerarse como elementos para indicación de cirugía temprana en pacientes asintomáticos ${ }^{(4)}$.

Más allá de la ausencia de estudios randomizados que comparen los resultados de reparación versus sustitución en IM primaria, es comúnmente aceptado que la reparación (duradera) es preferible sobre la sustitución valvular. Sin embargo, esa durabilidad está supeditada al tipo de mecanismo regurgitante, y es mayor en el prolapso posterior y menor en el prolapso anterior y bivalvar ${ }^{(5)}$.

Cuando la reparación no es posible o no resulta exitosa, debe procederse a la sustitución con conservación del aparato subvalvular. La conservación total de la válvula nativa es la cirugía predominantemente realizada en nuestro medio (la válvula no se sustituye; se implanta la prótesis dentro de la válvula nativa replegada dejando indemne el aparato subvalvular).

Según las guías europeas ${ }^{(4)}$, la cirugía debería ser considerada en pacientes asintomáticos con FEVI $>60 \%$ y DSVI: 40-44 mm cuando la válvula es reparable, el riesgo quirúrgico bajo y el paciente presente flail o dilatación de $\mathrm{AI}$ (indicación IIa C).

Los pacientes asintomáticos con FEVI > 60\% pueden ser seguidos con ETT cada seis meses, o más frecuentemente si aparecen cambios en las mediciones de FEVI y DSVI o se está cerca de los límites, ya que cuando la cirugía se realiza más allá de los dos meses posteriores a alcanzar estos umbrales se asocia con resultados posoperatorios adversos.

La paciente, sin embargo, estaría incluida en el grupo de asintomáticos con FEVI $\leq 60 \%$ (indicación I B de cirugía mitral, según Guías ESC). La FEVI 60\% ya representa un compromiso ventricular leve en este escenario. Adicionalmente se hace referencia a un ecocardiograma previo con FEVI $66 \%$.

Según la actualización 2017 de las guías americanas $2014^{(1)}$, los pacientes con IM severa primaria crónica, FEVI $30 \%$ a $60 \%$ o DSVI > $40 \mathrm{~mm}$ (estadio C2), o ambos, tienen indicación de cirugía sobre la válvula mitral (indicación I B). Esta recomendación no está apoyada en estudios randomizados.

Según esta guía, la indicación, en este subgrupo de pacientes, no está condicionada por la técnica a utilizar. La plastia valvular solo está recomendada en el prolapso exclusivo del velo posterior que no está presente en la paciente en cuestión.

Si concediéramos erróneamente incluir a la paciente en el grupo con FEVI $>60 \%$ y DSVI $<40 \mathrm{~mm}$ (estadio C1) se recomendaría cirugía de reparación mitral exclusivamente, y debería cumplirse el exigente requisito de estimar un éxito de la reparación $>95 \%$.

Sin embargo, según estos autores, en este grupo (estadio C1), es recomendable proceder a cirugía de reparación o sustitución si se presenta disminución de la FEVI o dilatación del VI, aun dentro de parámetros "normales" (FEVI > 60\% y DSVI <40 mm), ya que nos sitúa a la puerta del compromiso anátomo-funcional del VI (indicación IIa, nivel de evidencia C-LD). Esta paciente presentó descenso de la FEVI y aumento del DSVI, por lo que igualmente sería recomendable proceder con cirugía independientemente de que sea sustitución o reparación.

Esta última recomendación está basada en la fisiopatología irreversible de la IM. Si bien es variable el curso temporal, la sobrecarga de volumen y el aumento del estrés valvular determinan mayor insuficiencia, mayor dilatación y así sucesivamente. Un estudio de Tribouilloy, citado en esta guía, encontró que DSVI $>37 \mathrm{~mm}$ o FEVI <64\% preoperatorios son puntos de corte ya relacionados con compromiso del remodelado positivo posoperatorio.

En otro estudio no randomizado ${ }^{(6)}, 447$ pacientes consecutivos con IM primaria asintomática y FEVI $>60 \%$ fueron asignados según criterio médico a cirugía precoz o seguimiento conservador. Luego se realizó un propensity score matching. El $94 \%$ de los pacientes operados recibió plastia mitral. En el seguimiento a largo plazo la cirugía precoz se asoció con menor tasa de eventos, menor mortalidad cardíaca e internación por falla cardíaca, sugiriendo que la cirugía precoz sería una mejor opción en pacientes asintomáticos.

Otro resultado destacado fue que los pacientes que accedieron más tardíamente a cirugía tenían menor FEVI, mayor dilatación del VI y mayor mortalidad del procedimiento.

Está demostrado que incluso realizando reparación valvular, la clase funcional de la NYHA preoperatoria es un factor que influye de forma independiente y significativa en la sobrevida de pacientes que van a cirugía mitral(7).

Los pacientes operados en clases I y II tienen similar sobrevida que la población general de igual edad y 
sexo, pero esto no es así en los pacientes con clases III y IV (incluso en una población exclusiva de pacientes plastiados) ${ }^{(8)}$. Teóricamente esta diferencia sería mayor en el caso de realizar sustitución por ser un procedimiento de mayor mortalidad inicial.

\section{El dilema "reparación o sustitución"}

Es discutible qué volumen quirúrgico individual e institucional es necesario para mantener buenos resultados en reparación mitral. El mínimo anual por cirujano para conseguir resultados óptimos sería entre 20 a 40 cirugías, según diversos autores. Institucionalmente serían necesarios unos 50 casos/año. Otros criterios postulados son mortalidad operatoria $<1 \%$ y reintervención a cinco años $<5 \%{ }^{(9)}$

Según datos de Medicare 2000-2009, 91\% de los centros de Estados Unidos realizaron menos de 40 cirugías mitrales/año, y 94\% menos de 20 reparaciones mitrales/año ${ }^{(10)}$.

Esta realidad no es ajena a nuestro medio en el que promedialmente cada cirujano realiza 100 cirugías/año como primer operador. De estas, 30\%-40\% son valvulares y una minoría mitrales. De este último grupo, la mayoría de pacientes son referidos por su cardiólogo en etapas avanzadas de la enfermedad (indicaciones clase I). Esto desalienta a introducirse en la "aventura" de la reparación mitral en un paciente que habitualmente ya está en edad de recibir una prótesis biológica y presenta FEVI descendida, VI dilatado y menor "margen de maniobra" para intentar una plastia. En suma, todos contribuimos a que la mayoría de los pacientes reciban una prótesis.

\section{Conclusiones}

El timing óptimo de la cirugía mitral en IM primaria asintomática es discutible. La cirugía "precoz" no trata síntomas, pero provee una sobrevida igual a la de la población general a costa de un bajo riesgo.

La cirugía mitral "indicación clase I" trata síntomas predominantemente, pero ya está disminuida la sobrevida, aun luego de una cirugía sin complicaciones posoperatorias. En la concepción más moderna se definiría como una cirugía de rescate.

En cuanto a esta paciente, pensamos que tiene indicación actual de cirugía por el descenso de la FEVI y la dilatación leve del VI. Si bien está en el límite de ambos parámetros, la evolución reciente y la edad de la paciente alientan a cirugía precoz. Otros exámenes paraclínicos detallados podrían reafirmar más la indicación. Sería ideal restaurar la competencia valvular mediante plastia, aunque por las razones expuestas seguramente recibiría una prótesis mecánica y anticoagulación crónica.

\section{Bibliografía}

1. Nishimura RA, Otto CM, Bonow RO, Carabello BA, Erwin JP 3rd, Fleisher LA, et al. 2017 AHA/ACC Focused Update of the 2014 AHA/ACC Guideline for the Management of Patients With Valvular Heart Disease. A Report of the American College of Cardiology/American Heart Association Task Force on Clinical Practice Guidelines. J Am Coll Cardiol 2017;70(2):252-89. doi: 10.1016/j.jacc.2017.03.011

2. Magne J, Lancellotti P, Pierard L. Exercise pulmonary hypertension in asyntomatic degenerative mitral regurgitation. Circulation 2010;122(1):33-41 doi: 10.1161/CIRCULATIONAHA.110.938241.

3. Mehta NK, Kim J, Siden JY, Rodriguez-Diego S, Alakbarli J, Di Franco A, et al. Utility of cardiac magnetic resonance for evaluation of mitral regurgitation prior to mitral valve surgery. J Thorac Dis 2017; 9(Suppl 4): S246-6. doi: $10.21037 /$ jtd.2017.03.54.

4. Baumgartner H, Falk V, Bax JJ, De Bonis M, Hamm C, Holm PJ, et al. 2017 ESC/EACTS Guidelines for the management of valvular heart disease. Eur Heart J. 2017;38(36): 2739-91. doi: 10.1093/eurheartj/ehx391

5. David T, Ivanov J, Armstrong S, Christie D, Rakowski H. A comparison of outcomes of mitral valve repair for degenerative disease with posterior, anterior, and bileaflet prolapse. J Thorac Cardiovasc Surg 2005;130(5):1242-9.

6. Kang DK, Kim JH, Rim JH, Kim MJ, Yun SC, Song JM, et al. Comparison of early surgery versus conventional treatment in asymptomatic severe mitral regurgitation. Circulation 2009;119 (6):797-804. doi: 10.1161/CIRCULATIONAHA.108.802314.

7. David TE, Armstrong S, McCrindle BW, Manlhiot C. Late outcomes of mitral valve repair for mitral regurgitation due to degenerative disease. Circulation 2013;127 (14):1485-92. 1485-92. doi: 10.1161/CIRCULATIONAHA.112.000699.

8. David TE, Ivanov J, Armstrong S, Rakowski H. Late outcomes of mitral valve repair for floppy valves: Implications for asymptomatic patients. J Thorac Cardiovasc Surg 2003;125 (5):1143-52.

9. Chambers J, Ray S, Prendergast B, Graham T, Campbell B, Greenhalgh D, et al. Standards for heart valve surgery in a Heart Valve Center of Excellence. Open Heart 2015;2(1):e000216. doi: 10.1136/openhrt-2014-000216. eCollection 2015.

10. Vassileva CM, McNeely C, Spertus J, Markwell S, Hazelrigg S. Hospital volume, mitral repair rates, and mortality in mitral valve surgery in the elderly: an analysis of US hospitals treating Medicare fee-for-service patients. J Thorac Cardiovasc Surg 2015;149(3):762-8. e1 doi: 10.1016/j.jtcvs.2014.08.084. 


\section{Por qué seguimiento y control Dr. Gustavo Junker}

Considerando el caso clínico, se trata de una paciente de sexo femenino, joven, de 39 años, que se la conoce como portadora de una insuficiencia mitral asintomática.

Los parámetros ecocardiográficos muestran: diámetro sistólico de ventrículo izquierdo (DSVI): $44 \mathrm{~mm}$, diámetro de aurícula izquierda (AI): $40 \mathrm{~mm}$, fracción de eyección del ventrículo izquierdo (FEVI): 60\% y una válvula mitral definida como mixomatosa con prolapso de ambos velos, generando una insuficiencia severa. Partimos de la base que cumple con los criterios ecocardiográficos de severidad: vena contracta $\geq 0,7 \mathrm{~cm}$, volumen regurgitante $\geq 60 \mathrm{ml}$, fracción regurgitante $\geq 50 \%$, orificio regurgitante $\geq 0,4 \mathrm{~cm}^{2}$.

Una primera consideración a realizar es que se trata de lo que se denomina insuficiencia mitral primaria, por compromiso del propio aparato valvular, lo que la diferencia de las secundarias en donde la conducta y el pronóstico son diferentes, ya que se deben sobre todo a alteraciones de la geometría ventricular ${ }^{(1,2)}$.

Una segunda consideración es que estos pacientes, en los que existe discusión sobre cuál es la mejor conducta a adoptar, deben ser controlados y resueltos por lo que se denomina heart team o grupo cardiológico, si lo existiera, especializado en valvulopatías, compuesto por cardiólogo tratante, ecocardiografista, cirujano e intervencionista. La observación múltiple de profesionales entrenados en estas patologías es lo que, sin dudas, genera los mejores resultados.

Hechas estas aclaraciones pasaremos a ver cuál es la información con la que cuenta la cardiología clínica para definir la oportunidad quirúrgica de corrección de la insuficiencia valvular mitral.

Para ello, lo primero es definir la sintomatología, por lo que se describe, la paciente desarrolla su actividad sin disnea y se debe tratar de especificar un poco más qué capacidad funcional tiene realmente, con el interrogatorio apropiado, vinculándolo a su actividad domiciliaria o de trabajo e incluso con eventual ejercicio aeróbico. Si eso no pudiera establecerse claramente, podemos realizar una prue- ba ergométrica graduada. Existen publicaciones, como la de Supino y colaboradores ${ }^{(3)}$, donde se demuestra que la duración del ejercicio, la frecuencia cardíaca alcanzada, y la frecuencia cardíaca en la recuperación son predictores de proximidad de la cirugía. Aquellos que no toleran 15 minutos de ejercicio, o no llegan al 99\% de la frecuencia cardíaca máxima prevista y no tienen una recuperación de 29 latidos en el minuto, son los pacientes en que se debe indicar la cirugía.

Otra herramienta con la que contamos en pacientes asintomáticos es la medición del BNP o péptido natriurético cerebral, hormona secretada por las células miocárdicas en respuesta al estrés diastólico por sobrecarga de volumen.

Existen dos trabajos que demostraron su utilidad en la insuficiencia mitral severa asintomática, uno de 2009, de un grupo argentino ${ }^{(4)}$ que demostró que un valor de corte de $\mathrm{BNP} \geq 105 \mathrm{pg} / \mathrm{ml}$ permite discriminar a un subgrupo de pacientes con mayor riesgo de desarrollar disfunción ventricular izquierda clínica y ecocardiográfica, con descenso de la fracción de eyección, y otro de $2016^{(6)}$, que comprobó que en pacientes asintomáticos con FEVI normal, el BNP es un predictor independiente de eventos cuando los valores absolutos superan los $60 \mathrm{pg} / \mathrm{ml}$.

En lo que concierne a los parámetros ecocardiográficos debemos hacer algunas consideraciones sobre las repercusiones que ha generado la insuficiencia mitral sobre la función ventricular y diámetros de cavidades en esta paciente.

Las últimas guías publicadas este año, tanto de la Sociedad Europea de Cardiología (ESC), como de la Sociedad Americana del Corazón con el Colegio Americano del Corazón (AHA-ACC), concuerdan en que el límite para considerar la cirugía en pacientes asintomáticos es una FEVI de $60 \%$.

Lo anterior está basado, entre otros, en el trabajo de Rosenhek y colaboradores ${ }^{(5)}$, y de Enriquez-Sarano y colaboradores de 1994(6). En el primero de ellos se demostró que los pacientes asintomáticos con FEVI >60\%, sin hipertensión pulmonar ni fibrilación auricular y con diámetros que

Cardiólogo. Asociación Española, SMI, CASMU. Correspondencia: Dr. Gustavo Junker.

Correo electrónico: gjunkerli@gmail.com

Recibido Oct 18, 2017; aceptado Nov 6, 2017 
analizaremos, pueden ser controlados periódicamente con muy buenos resultados y con períodos prolongados libres de eventos.

En el segundo, en el que se controlaron 409 pacientes operados por insuficiencia mitral severa, comprobaron una marcada mortalidad en los mayores de 75 años y en los que tenían FEVI <60\%. Los resultados mostraron que en un seguimiento a diez años para FEVI $\geq 60 \%$, la sobrevida es de $72 \%$; FEVI entre $50 \%$ y $60 \%$, sobrevida de $53 \%$, y para FEVI $<50 \%$, sobrevida de $32 \%$, de tal manera que ambos permitieron establecer, y así aparece en las guías, que la FEVI límite para definir la cirugía es de $60 \%$.

Otro elemento a considerar son los diámetros del VI. En este punto existen diferencias entre la AHA-ACC, que establece como límite máximo de DSVI: $40 \mathrm{~mm}$ y la ESC que, por su parte, considera un DSVI: $45 \mathrm{~mm}$.

Lo establecido por AHA-ACC se basa en el trabajo de Tribouilloy y colaboradores ${ }^{(7)}$, en el que la sobrevida a diez años con conducta conservadora (sin cirugía) cuando el DSVI $<40 \mathrm{~mm}$ es de $64 \pm 5 \%$ versus $48 \pm 10 \%$ con un DSVI $\geq a 40 \mathrm{~mm}$. Un valor $\geq$ $40 \mathrm{~mm}$ es predictor independiente de mortalidad total y cardíaca. Cuando se realiza cirugía con diámetros mayores se produce un exceso de mortalidad, tanto para muerte total (hazard ratio [HR] 1,86, IC 95\%: 1,11-3,15), como para muerte cardíaca (HR 1,81, IC 95\%: 1,05-3,54).

Las Guías ESC establecen como punto de corte $45 \mathrm{~mm}$ de DSVI, fundamentalmente para el prolapso de válvula mitral.

Otro de los parámetros ecocadiográficos predictores de eventos es la medición de la presión sistólica en la arteria pulmonar (PSAP), aunque no contamos con este dato en nuestra paciente.

En el trabajo de Ghoreishi y colaboradores de $2011^{(8)}$ se demuestra que hay una correlación directa entre la mortalidad operatoria y la PSAP. Cuando la PSAP es < $40 \mathrm{mmHg}$ la mortalidad es de $2 \%$; con una PSAP de $40-50 \mathrm{mmHg}$, mortalidad $3 \%$; PSAP de $50-60 \mathrm{~mm} \mathrm{Hg}$, mortalidad $8 \%$ y valores mayores de 60 mmHg de PSAP se correlacionan con una mortalidad de $12 \%$, siendo la sobrevida a cinco años de $88 \%, 79 \%$, $65 \%$ y $53 \%$, respectivamente. Por tanto, la PSAP es un poderoso predictor de sobrevida precoz y tardía en la cirugía de la insuficiencia mitral.

También, si existieran dudas, se puede medir en el ejercicio, como en el trabajo de Magne y colaborado$\mathrm{res}^{(9)}$. De los pacientes que llegan a $60 \mathrm{mmHg}$ de PSAP en ejercicio solo $35 \% \pm 8$ están libres de eventos a dos años versus $75 \pm 7 \%$, ( $<<0,0001)$ en los que no la desarrollan, (HR 2,8, IC 95\%: 1,4-5,4, p=0,002).

En el caso que se presenta se debería considerar la ecocadiografía transesofágica, que junto con la imagen en 3D permiten una mejor definición anátomo-funcional del prolapso valvular, observándose cuáles velos y festones compromete, lo que permite establecer con mayor certeza el grado de reparabilidad de la válvula y determinar la mejor estrategia quirúrgica. La reparabilidad es mayor y más duradera cuando existe compromiso exclusivo de la valva posterior, sobre todo en su sector central (P2) que la bivalvar como se consigna en esta paciente.

Finalmente, hay otros dos elementos a considerar, el diámetro de la AI y el volumen auricular izquierdo.

En el trabajo de Le Tourneau y colaboradores $^{(10)}$, con seguimiento de 492 pacientes, observaron que el volumen indexado de la $\mathrm{AI}$ es un indicador de aumento de eventos adversos, de tal manera que los que tienen un volumen indexado $\geq 60 \mathrm{ml} / \mathrm{m}^{2}$ tienen menos sobrevida a cinco años que frente a un volumen menor $(53 \pm 8,6 \%$ versus $76 \%$ respectivamente, $p=0,017$ ). La mortalidad se incrementa por 2,8 veces y los eventos cardíacos por 5,2 veces cuando reciben tratamiento médico. La cirugía mitral en estos pacientes se asoció con disminución de la mortalidad y de eventos cardíacos (HR 0,46, IC 95\%: 0,26-0,84, p=0,01) y (HR 0,38, IC 95\%: $0,23-0,62, p=0,0001)$. Cabe agregar que hay estudios de medición de la función auricular, como la fracción de vaciado auricular, utilizando imagen en 2D con speckle, y con método de Simpson (máximo volumen auricular vs mínimo).

En esta paciente se hace referencia a un diámetro de AI: $40 \mathrm{~mm}$, que si bien no es una medida volumétrica, se puede considerar normal.

El último elemento clínico de relevancia es la presencia de fibrilación auricular. El registro del seguimiento longitudinal de las reparaciones mitrales publicado en 2012 de la Sociedad Americana de Cirujanos de Tórax, realizado entre 1997 y 2007 sobre 14.604 reparaciones en mayores de 65 años, muestra que la fibrilación auricular es un poderoso predictor de reingreso por falla cardíaca a los cinco años (HR 1,51, IC 95\%: 1,39-1,66).

En principio consideramos que esta paciente presenta ritmo sinusal.

Las Guías ESC aconsejan cirugía como indicación IIa (debería ser considerada), nivel de evidencia $\mathrm{C}$ (consejo de expertos o pequeños registros) en pacientes asintomáticos, cuando hay alta probabilidad de reparación durable, en centros especializados con muy baja mortalidad.

La Guía AHA-ACC establece la cirugía como indicación IIa (razonable) nivel de evidencia B (moderada calidad de evidencia de un estudio clínico randomizado) en pacientes asintomáticos, cuando la probabilidad de éxito y duración de la reparación 
sin insuficiencia mitral residual es mayor a $95 \%$ y en centros con mortalidad operatoria esperada menor a $1 \%$.

Incluye, además, como indicación IIa, nivel de evidencia C-LD (estudios observacionales o registros) a pacientes asintomáticos con progresivo incremento del tamaño del VI o disminución de la FEVI en estudios seriados.

Es de destacar que tanto los registros americanos como los europeos estiman en cerca de $60 \%$ las reparaciones y solo en centros muy especializados se llega a $95 \%$.

Dadas todas las consideraciones realizadas y que se trata de una paciente asintomática sin ningún parámetro ecocardiográfico indicador de oportunidad quirúrgica actual: FEVI normal, sin dilatación de cavidades, hipertensión pulmonar ni fibrilación auricular, parece razonable adoptar una conducta de observación y seguimiento clínico con ecocardiografía seriada cada tres a seis meses.

Con este seguimiento se podrá establecer apropiadamente el momento oportuno para intervenirla quirúrgicamente.

\section{Bibliografía}

1. Baumgartner H, Falk V, Bax JJ, De Bonis M, Hamm C, Holm PJ, et al. 2017 ESC/EACTS Guidelines for the management of valvular heart disease. Eur Heart J 2017;38(36): 2739-86 doi: 10.1093/eurheartj/ehx391

2. Nishimura RA, Otto CM, Bonow RO, Carabello BA, Erwin JP 3rd, Fleisher LA, et al. 2017 AHA/ACC focused update of the 2014 AHA/ACC guideline for the management of patients with valvular heart disease: a report of the American College of Cardiology / American Heart Association task force on clinical practice guidelines. Circulation 2017; 135(25):e1159-95. doi: 10.1161/CIR.0000000000000503.)

3. Supino PG, Borer JS, Schuleri K, Gupta A, Hochreiter C, Kligfield P, et al. Prognostic value of exercise tolerance testing in asymptomatic chronic nonischemic mitral regurgitation. Am J Cardiol 2007;100(8):1274-81.

4. Pizarro R, Bazzino OO, Oberti PF, Falconi M, Achilli F, Arias A, et al. Prospective validation of the prognostic usefulness of brain natriuretic peptide in asymptomatic patients with chronic severe mitral regurgitation. J Am Coll Cardiol 2009;54(12) :1099-106. doi: 10.1016/j.jacc.2009.06.013.

5. Rosenhek R, Rader F, Klaar U, Gabriel H, Kreje M, Kalbeck D, et al. Outcome of watchful waiting in asymptomatic severe mitral regurgitation. Circulation 2006;113(18):2238-44.

6. Enriquez-Sarano M, Tajik AJ, Schaff HV, Orszulak TA, Bailey KR, Frye RL. Echocardiographic prediction of survival after surgical correction of organic mitral regurgitation. Circulation 1994;90(2):830-7.

7. Tribouilloy C, Grigioni F, Avierinos JF, Barbieri A, Rusinaru D, Szymanski C, et al. Survival implication of left ventricular end-systolic diameter in mitral regurgitation due to flail leaflets. J Am Coll Cardiol 2009;54:1961-8. doi: 10.1016/j.jacc.2009.06.047.

8. Ghoreishi M, Evans CF, DeFilippi CR, Hobbs G, Young CA, Griffith BP, et al. Pulmonary hypertension adversely affects short- and long-termsurvival after mitral valve operation for mitral regurgitation: Implications for timing of surgery. J Thorac Cardiovasc Surg 2011;142(6):1439-52.

doi: 10.1016/j.jtcvs.2011.08.030

9. Magne J, Lancellotti P, Piérard LA. Exercise pulmonary hypertension in asymptomatic degenerative mitral regurgitation. Circulation 2010;122(1): 33-41. doi: 10.1161/CIRCULATIONAHA.110.938241.

10. Le Tourneau T, Messika-Zeitoun D, Russo A, Detaint D, Topilsky Y, Mahoney DW, et al. Impact of left atrial volume on clinical outcome in organic mitral regurgitation. J Am Coll Cardiol 2010;56(7):570-8. doi: 10.106/j.jacc.2010.02.059

Respuesta al Dr. Gustavo Junker

\section{La opción continúa siendo cirugía precoz}

\section{Introducción}

En primer lugar destaco que tenemos varias referencias bibliográficas en común y las restantes tratan sobre los mismos puntos para ambas posturas. Esto facilita la discusión y evita un eventual sesgo de búsqueda. Sin embargo, se analizó la misma evidencia dándole un significado diferente, lo que hace aún más rica la discusión sobre la mejor opción tera- péutica, la cual ciertamente debe ser encarada como heart team.

\section{Qué proponemos en común}

Se postuló una evaluación complementaria similar, considerando que faltan datos básicos para definir indicación quirúrgica o seguimiento. Más allá de la 
evaluación por imagen, a la que agregaríamos una resonancia cardíaca, el colega destacó elementos de suma importancia como la determinación de la presión sistólica arterial pulmonar (PSAP) en el esfuerzo y una prueba de ejercicio para evaluar la tolerancia al mismo como elemento pronóstico. Se suma además a la evaluación la dosificación del péptido natriurético BNP.

Sin embargo, en nuestro rol impuesto de defensor de la conducta quirúrgica precoz, seguiremos analizando el caso según los datos aportados hasta el momento.

\section{Cuáles son nuestras diferencias}

El Dr. Junker analiza la indicación según las recomendaciones de las guías americanas y europeas ${ }^{(1,2)}$, tal cual lo hicimos nosotros, girando inicialmente en torno a la fracción de eyección. En ambas guías la cirugía está indicada (indicación clase I) en los pacientes con fracción de eyección del ventrículo izquierdo (FEVI) $30 \%$ a $60 \%$, categoría en la que está incluida esta paciente. Es cierto que los pacientes con FEVI > $60 \%$ tienen mejor pronóstico y podrían ser seguidos con un control muy estrecho, pero esta paciente ya alcanzó dicho umbral.

Esta FEVI de 60\% es “pseudonormal”. Sistemáticamente vemos en el posoperatorio no complicado de cirugía por insuficiencia mitral (IM), una disminución promedial de $10 \%$ en el valor absoluto de FEVI. Groseramente se podría estimar que la FEVI "real” de esta paciente al eliminar la IM es de 50\%.

Afortunadamente está en el límite y aún es "poco clara" esta indicación clase I.

He aquí uno de los conceptos fundamentales que queremos remarcar: cuanto más seguros estemos de enviar a un paciente a cirugía mitral, peor pronóstico tendrá el mismo.

Se analiza un importante trabajo de M. Enríquez-Sarano ${ }^{(3)}$ en el que queda demostrado que pequeñas caídas de la FEVI determinan enormes impactos en el pronóstico de los pacientes con IM. Si la FEVI es $>60 \%$ la sobrevida a diez años es $72 \%$, cae a $53 \%$ para FEVI 50\% a $60 \%$ (como en esta paciente de 39 años) y a $32 \%$ de sobrevida a diez años si la FEVI es $<50 \%$. Este trabajo se condice con uno similar de Tirone David que presentamos en la sección inicial(4).

Posteriormente se considera el trabajo de Tribouilloy ${ }^{(5)}$ para analizar los diámetros ventriculares de pacientes que se benefician de cirugía. Allí queda claramente establecido que un diámetro sistólico de VI (DSVI) $>40 \mathrm{~mm}$ se asocia a una menor sobrevida a 10 años. Asimismo, cuando se realiza cirugía una vez alcanzado este diámetro, no solo la mortalidad del procedimiento es mayor, sino que la sobrevida posoperatoria es menor que en los pacientes con menor diáme- tro ventricular. Esto, si se interpreta correctamente, reafirma la idea de que los pacientes que dilatan su ventrículo, próximo o más allá de estos límites, deben operarse precozmente, de lo contrario presentan peor pronóstico.

$\mathrm{Al}$ respecto de las dos indicaciones que se analizan de cirugía de reparación, con alta probabilidad de alcanzar la misma y baja mortalidad, creemos que se refieren a otro subgrupo de pacientes al que no pertenece la paciente. Se cita, por ejemplo, la indicación de la guía americana "II a, nivel de evidencia B, en pacientes asintomáticos, cuando la probabilidad de reparación es mayor a 95\% y en centros con mortalidad esperada $<1 \%$ ". Esta indicación se refiere a pacientes con IM asintomática, que además tengan FEVI $>60 \%$, DSVI $<40 \mathrm{~mm}$, ausencia de hipertensión pulmonar y fibrilación auricular. Según la misma guía, esta paciente tiene indicación clase I de cirugía por FEVI $60 \%$ por un lado y por DSVI > $40 \mathrm{~mm}$ por otro.

Le damos la derecha a nuestro compañero de controversia al respecto del bajo porcentaje comunicado de reparaciones mitrales, siendo los números internacionales que presentamos en la sección anterior incluso mucho menos alentadores, realidad que no escapa a la de nuestro medio. Sin embargo, repetimos que realizar o no plastia valvular, que puede ser beneficiosa en ciertos escenarios, no determina la indicación de cirugía en esta paciente.

Por último, disentimos en que no presenta compromiso de la FEVI ni dilatación del VI, argumentos por los cuales se llega a la conclusión de que no presenta indicación quirúrgica. Como ya fue postulado, un descenso de la FEVI a $60 \%$ o menos, en el contexto de IM severa, se considera compromiso de la función contráctil del VI y se asocia a compromiso de la sobrevida, incluso de mediar un tratamiento de la valvulopatía. Por otro lado, las guías americanas consideran un DSVI >40 mm como asociado a peores resultados en el seguimiento (recordamos que presenta $44 \mathrm{~mm}$ ), mientras las europeas toman como umbral $45 \mathrm{~mm}$ (reconozco que le falta $1 \mathrm{~mm}$ ).

\section{"Guías son guías" y "los números no son todo"}

No debemos perder de vista que estamos frente a una paciente muy joven, aspecto muy importante a tener en cuenta para defender cualquiera de las dos conductas, aunque continuaré argumentando a favor de la que me fue asignada.

Las guías contienen elementos orientadores, y están basadas en ensayos grandes o pequeños, randomizados o no, pero principalmente en evidencia observacional, opinión de expertos, etc. Esto les da cierta "debilidad", o desde un punto de vista más optimista, de flexibilidad para su interpretación e instrumentación. 
Gran parte de la discusión aquí planteada se desvanecería si la paciente presentara FEVI 59\% o FEVI $61 \%$. Ambos valores, muy próximos, se encuentran incluso dentro de la variabilidad inter e intraobservador.

Desde nuestra experiencia profesional consideramos (con una alta probabilidad, según los trabajos presentados en la sección inicial) que esta paciente se tornará sintomática en los próximos tres años. Dado el mecanismo intrínseco de la IM y el estado actual en esta paciente, considero muy probable que deteriore aún más la FEVI o dilate más el VI en el corto a mediano plazo. Una vez que esto ocurra, como está demostrado, su pronóstico vital posoperatorio estará considerablemente comprometido. Este pasaje al estado sintomático (indicación clase I de cirugía) habitualmente se acompaña de más descenso de la FEVI (actualmente ya jaqueada).

Ya vimos que ante un descenso "leve" de la FEVI, por ejemplo a 55\%, la sobrevida estará muy comprometida (todo esto en una paciente de 39 años). De nuevo, cuanto más seguros estemos de enviar a cirugía a nuestros pacientes, peor será su pronóstico posoperatorio. Que esta conducta cambie forma parte de la "historia natural de las indicaciones" en la cirugía cardíaca, una especialidad relativamente nueva, que al disminuir constantemente su morbimortalidad en las últimas décadas ha ampliado su campo de acción a pacientes más enfermos, y también a pacientes más sanos, al ofrecer una mejor relación riesgo-beneficio.

Pero la decisión no es tan fácil, reconozcamos qué hay del otro lado de la balanza. Principalmente existen dos elementos negativos: mortalidad operatoria (MO) y enfermedad asociada a la prótesis valvular cardíaca. La MO en una paciente de estas características (aunque faltan datos para determinar correctamente el riesgo operatorio) sería muy baja en nuestro

\section{Bibliografía}

1. Nishimura RA, Otto CM, Bonow RO, Carabello BA, Erwin JP 3rd, Fleisher LA, et al. 2017 AHA/ACC Focused update of the 2014 AHA/ACC Guideline for the management of patients with valvular heart disease: a report of the American College of Cardiology/American Heart Association Task Force on Clinical Practice Guidelines. J Am Coll Cardiol 2017;70(2):252-89.doi: 10.1016/j.jacc.2017.03.011.

2. Baumgartner H, Falk V, Bax JJ, De Bonis M, HammC, HolmPJ, et al. 2017 ESC/EACTS Guidelines for the management of valvular heart disease. Eur Heart J 2017;38(36): 2739-91. doi: 10.1093/eurheartj/ehx391.

3. Enriquez-Sarano M, Tajik AJ, Schaff HV, Orszulak TA, Bailey KR, Frye RL. Echocardiographic prediction of survival after surgical correc- medio, incluso realizando sustitución valvular. Podríamos estimarla entre $1 \%$ a $2 \%$. Este riesgo es una fracción comparada con la pérdida de sobrevida que presentan los pacientes que se vuelven sintomáticos o que asocian mayor descenso de la FEVI.

El segundo punto, más importante que el anterior, es la enfermedad protésica, especialmente endocarditis infecciosa (EI), trombosis valvular y eventos hemorrágicos.

Estas tres complicaciones no estarán presentes de poder realizar una plastia exitosa. Sin embargo, es altamente probable que en nuestro medio reciba una válvula protésica.

Dada la sobrevida esperada, la prótesis deberá ser mecánica y anticoagularse crónicamente. Podría recibir una válvula biológica por voluntad de la paciente (por desear otro embarazo, por ejemplo) sabiendo que la reintervención de la válvula mitral constituye una cirugía de alto riesgo.

La incidencia de EI es muy baja (aproximadamente $0,3 \%$ anual en posición mitral) a diferencia de lo que ocurre en posición aórtica.

La incidencia de eventos tromboembólicos o sangrados mayores también es baja y para prevenirlos es de gran importancia el estricto control de la anticoagulación $^{(6)}$. Independientemente de esto y dada la edad de la paciente, de realizarse control trimestral, creemos improbable que llegue a intervenirse en edad de recibir una prótesis biológica.

Para finalizar, consideramos que con control trimestral o semestral, en una paciente de 39 años, tenemos poco que ganar: posponer algún tiempo indeterminado la cirugía, y mucho que perder: eventuales años de vida en una paciente, que según la expectativa de vida de la mujer en Uruguay tiene por delante varias décadas una vez resuelta su patología cardíaca.

\section{Dr. Gerardo Soca}

tion of organic mitral regurgitation. Circulation 1994;90(2):830-7.

4. David TE, Armstrong S, McCrindle BW, Manlhiot C. Late outcomes of mitral valve repair for mitral regurgitation due to degenerative disease. Circulation 2013;127 (14):1485-92. 1485-92. doi: 10.1161/CIRCULATIONAHA.112.000699.

5. Tribouilloy C, Grigioni F, Avierinos JF, Barbieri A, Rusinaru D, Szymanski C, et al. Survival implication of left ventricular end-systolic diameter in mitral regurgitation due to flail leaflets a long- term follow-up multicenter study. J Am Coll Cardiol 2009;54 (21):1961-8. 1961-8. doi: 10.1016/j.jacc.2009.06.047.

6. Pibarot $\mathbf{P}$, Dumesnil JG. Prosthetic heart valves: selection of the optimal prosthesis and long-term management. Circulation 2009; 119(7):1034-48. doi: 10.1161/CIRCULATIONAHA.108.778886. 


\section{Respuesta al Dr. Gerardo Soca}

Con respecto al planteo de la cirugía profiláctica sobre la válvula mitral, debemos realizar las siguientes consideraciones.

La cirugía valvular mitral de reemplazo tiene una mortalidad, según la Sociedad de Cirujanos de Tórax Americana, cercana a 5\% - 6\%, mientras que la reparación, que es muy dependiente de la experiencia del cirujano actuante, oscila entre $1 \%$ y $2 \%$.

La sobrevida al año es de 97,8\% en las reparaciones realizadas por cirujanos con más de 50 cirugías mitrales al año, mientras que es de $94,1 \%$ en los que intervienen menos de diez pacientes anuales. El porcentaje de reparaciones varía, según el volumen de operaciones de cada cirujano, entre $48 \%$ y $77 \%$. Esto está claramente demostrado en el trabajo publicado recientemente por los cirujanos del estado de Nueva York en JACC(1), que concluye que el volumen individual de cada cirujano es determinante no solo de las probabilidades de reparación, sino también del período libre de reintervención y de la sobrevida de los pacientes.

Pero veamos la realidad en nuestro medio, lo que nos va a permitir adoptar una mejor resolución del caso.

Obtuvimos datos de uno de los centros de alto volumen de intervenciones, que realiza aproximadamente 500 cirugías cardíacas anuales (se aproxima a la media de los otros centros). En un período de tres años se efectuaron 79 cirugías mitrales puras entre las cuales 45 correspondieron a sustitución mitral, con una mortalidad de 8,88\%, y 34 fueron plastias valvulares, lo que corresponde a $43 \%$ de los procedimientos, con una mortalidad de 5,88\%. Si bien es posible considerar estas cifras como aceptables para nuestro medio, distan de las recomendaciones de las guías, por lo que deben ser tenidas en cuenta al decidir una "cirugía precoz o profiláctica".

Además, de acuerdo al informe de la ecocardiografía, en nuestro caso el prolapso es bivalvar, lo que lleva a pensar que es muy probable que requiera de una sustitución por prótesis mecánica, lo que agrega la morbimortalidad del tratamiento anticoagulante a largo plazo.

También se debe tener en cuenta la posibilidad de una nueva gestación, lo que dificultaría el manejo de la anticoagulación crónica por los riesgos que implica el uso de warfarina durante el embarazo, única droga aceptada para las prótesis mecánicas, que puede conducir a graves complicaciones materno-fetales.

Por lo expuesto, planteamos continuar con una estrecha vigilancia de la paciente, para evitar la cirugía “tardía”, y creemos que en la situación clínica actual debemos mantener una conducta conservadora con vigilancia clínica y ecocardiográfica periódicas hasta la definitiva aparición de síntomas o alteraciones ecocardiográficas que nos indiquen la oportunidad quirúrgica.

Dr. Gustavo Junker

1. Chikwe J, Toyoda N, Anyanwu AC, Itagaki S, Egorova NN, Boateng $P$, et al. Relation of mitral valve surgery to repair rate, durability and survival. pii: S0735-1097(17)30677-0.

doi: 10.1016/j.jacc.2017.02.026. [Epub ahead of print] 